\title{
Atypical presentation and clinical management of jaundice secondary to Choledocoduodenal fistula: A Case Report
}

\author{
Smit Shah ${ }^{1}$, Dr. Praful Shah ${ }^{2}$
}

${ }^{1}$ MS-3, Rutgers Robert Wood Johnson Medical School, NJ, USA. ${ }^{2}$ M.S. General Surgeon, Fellow of Minimal Access Surgery, India.

\section{Abstract}

Choledocoduodenal fistula (CDDF) is an abnormal connection between the common bile duct and the duodenum which bypasses the normal opening of Ampulla of Vater. In this case, we describe the initial clinical presentation, diagnosis and treatment of a patient with CDDF along with prophylactic surgical management in order to prevent future hepatobiliary complications. In addition, we also discuss various risk factors of CDDF. Finally, we also discuss different types of CDDF along with its various hematologic and radiologic findings.

Keywords: Splenic, Hemoperitoneum, Colles, Blunt, Abdominal.

\section{Introduction}

Choledocoduodenal fistula (CDDF) is an abnormal connection between the common bile duct and the duodenum which bypasses the normal opening of Ampulla of Vater. Patients can present with '... abdominal pain, nausea, weight loss, diarrhea, and dyspeptic symptoms'. This in turn can also lead to 'malabsorption secondary to alteration in normal enterohepatic circulation of bile acid which can stimulate the colonic mucosa directly to secrete water and electrolytes excessively, leading to steatorrhea and diarrhea'. ${ }^{2}$ In this paper, we present a case of patient who had atypical presentation of CDDF with jaundice secondary to fibrosis of the Ampulla of Vater which led to fistula between the common bile duct and the duodenum, its surgical management along with patient's recovery after treatment.

\section{Case Details}

Patient is a 52-year-old female who presented to office with severe obstructive jaundice $\&$ abdominal pain since the past few months. Patient has a history of recurrent jaundice which was treated in a rural medical center of India with cholestyramine and IV fluids without any symptomatic relief 6 months prior to admission. Past medical history: as mentioned above. The patient denies any current medications, smoking, drinking or illicit drug use

\section{Examination}

On physical exam, patient had diffuse abdominal tenderness, icteric sclera, mild ascites and pallor.

\section{Investigative Reports}

On further evaluation, complete blood work was ordered along with liver function tests. Patient was found to be anemic with hemoglobin of $6.2 \mathrm{~g} / \mathrm{dl}$ along with elevated liver enzymes: AST: 150, ALT: 110 and WBC: $13000 \mathrm{~g} /$ dl. Total Bilirubin: 8, Direct Bilirubin: 4.9, Indirect Bilirubin: 3.1.

\section{Interventions and outcome}

Anemia was successfully treated initially with packed RBCs transfusion along with starting iron supplements. It took 12 days to get back to normal value of 12.1 .

CXR (Chest X-Ray), abdominal ultrasound and CT (Computerized Tomography) abdomen. CXR was negative but abdominal ultrasound (US) and CT abdomen demonstrated poor visualization of gall bladder along with few calculi and multiple well defined non-homogenously enhancing hypodense lesions in left and right lobe of the liver with air in biliary tree.

Consequently a diagnosis of multiple cholangitic abscess was made and patient was started on IV cefepime to resolve the infection. After this, patient was scheduled for ERCP (Endoscopic Retrograde Cholangiopancreatography) with stenting to relieve the jaundice. But since the CBD (Common Bile Duct) opening was not negotiated due to fibrosis of duodenal opening of ampulla and CBD. Therefore, stenting was performed through choledocoduodenal fistula following stone passage. 96 hours after stenting, patient underwent a prophylactic laparoscopic cholecystectomy followed by stent removal 48 hours after surgery. Patient had complete resolution of jaundice on 1 month status post laparoscopic cholecystectomy and stenting.

\section{Discussion}

CDDF has many different presentations ranging from symptoms of gastric outlet obstruction \& peptic ulcer disease (PUD) to common bile duct (CBD) obstruction $\&$ pneumobilia along with jaundice. In literature review, we found that majority of patients had CDDF secondary to perforation from duodenal ulcers which spontaneously form a fistulomatous connection between the duodenum and common bile duct

Correspondence: Smit Shah; 112 Montgomery Street, Apt 3G, Highland Park, NJ 08904, USA. E-mail: spshah1991@gmail.com. 
bypassing the Ampulla of Vater. ${ }^{3}$ However, here we present a case of patient who had atypical and unusual presentation of jaundice secondary to fibrosis of the ampulla which led to fistula between the CBD and the duodenum. In addition to PUD and distal fibrosis of ampullary opening, there are many different causes of CDDF like malignant neoplasms and cholelithiasis which can cause distal obstruction of CBD, in turn increasing pressure within the CBD leading to abnormal dilatation which results in fistulae tract between duodenum and $\mathrm{CBD} .^{3}$

From the perspective of pathophysiology, most common organs affected with CDDF are gallbladder and cystic duct. ${ }^{4}$ Furthermore, there is increased pressure within these organs. Due to limited space in the abdominal cavity, there is additional obstruction due to inferior movement of the gall bladder leading to compression of local vasculature which results in localized hypo-perfusion predisposing to necrosis. This increases the chances of multiple cholecystic abscess along with abnormal connections between gall bladder and small intestine also known as gall stone ileus which classically presents with pneumobilia and gall stone in the terminal ileum. One of the possible etiologies for cholangitic abscesses in this patient could be ascending infection from the duodenum into the CDDF into the bile duct into gall bladder parenchyma. Interestingly, recently there has been a recent case report of CDDF status post laparoscopic Duodeno-Ileal Switch which was successfully managed with 'conservative treatment including feeding and prolonged antibiotic treatment'. There are two types of distal CDDFs: Type 1 tends to be smaller in size secondary to smaller gall stones entering the intramural portion of the duodenum leading to fistula adjacent to the longitudinal fold along with duodenal papillae; and Type 2 tends to be bigger due to impaction of large stone on the extramural portion of CBD leading to fistula proximal and adjacent to duodenal fold. ${ }^{6-8}$

In our case, we can see that patient had multiple abscesses in and around the gall bladder leading to its poor visualization along with pneumobilia (Image A). Due to this, it was very difficult for us to decipher if patient had concurrent gall stones along with cholangitic abscesses and fistula. At this point, there was a broad list of differentials which included malignancy, infectious etiology or even gall stone ileus. However, it was the ERCP (Image B) that helped us to diagnose and confirm that patient had a CDDF leading to ascending cholangitis which could explain the presentation of multiple abscesses in gall bladder fossa. Due to heavy fibrosis and occlusion of ampullary opening, the decision was made to stent the CBD from the CDDF and in turn drain any gall stones if they were present in gall bladder.

Reason why this patient underwent a prophylactic laproscopic cholecystectomy is two-fold: ${ }^{7}$ (1) prevent future gall stones and prevent future obstruction and decrease chances of CDDF or gall stone ileus; and (2) Prevent additional proliferation of cells in the gall and decreasing the risk of cholangiocarcinoma. From

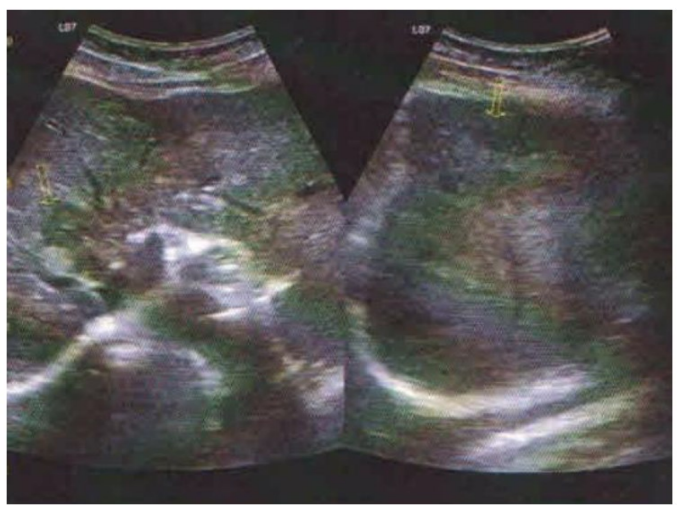

Figure 1: Ultrasound image on left demonstrates hypodense abscesses surrounding the porta with poor visualization of the gall bladder (Fluid collection in gall bladder fossa). Image on right shows mild presence of air in the biliary tree. Also (not shown in this image) is the present of peri-portal and para aortic lymph nodes which raised the suspicion of malignancy but was eventually ruled out.
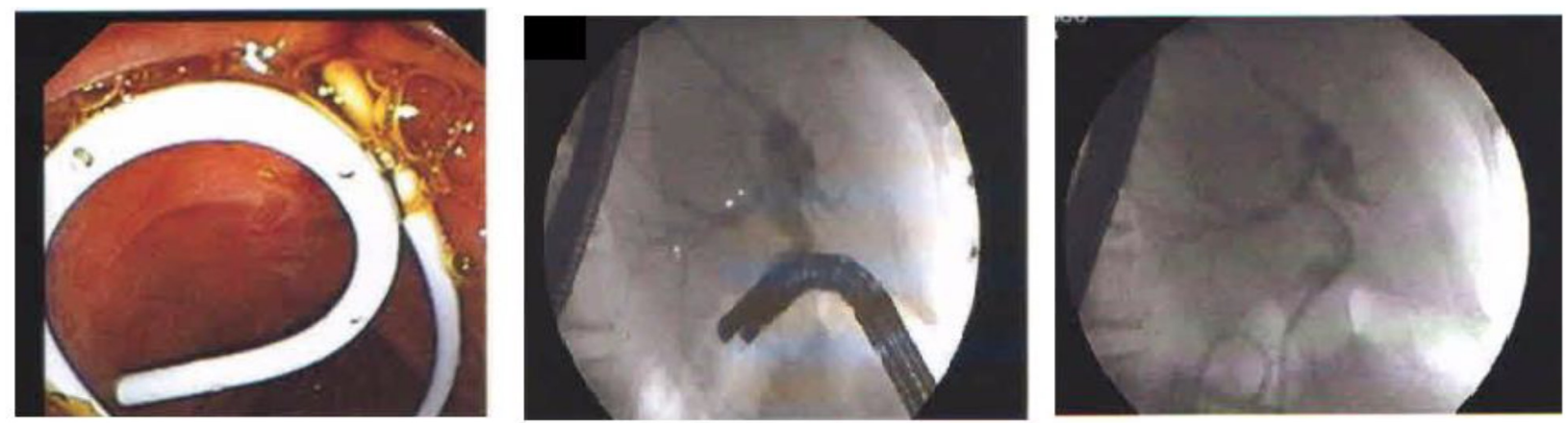

Figure 2: ERCP images above representing the choledocoduenotal fistula that was found during ERCP with stenting. In the middle we can see mildly dilated common bile duct $6 \mathrm{~mm}$. Plastic double pigtail stent was placed through the opening of the fistula.

South East Asia Journal of Public Health 2017;7(1):51-57 

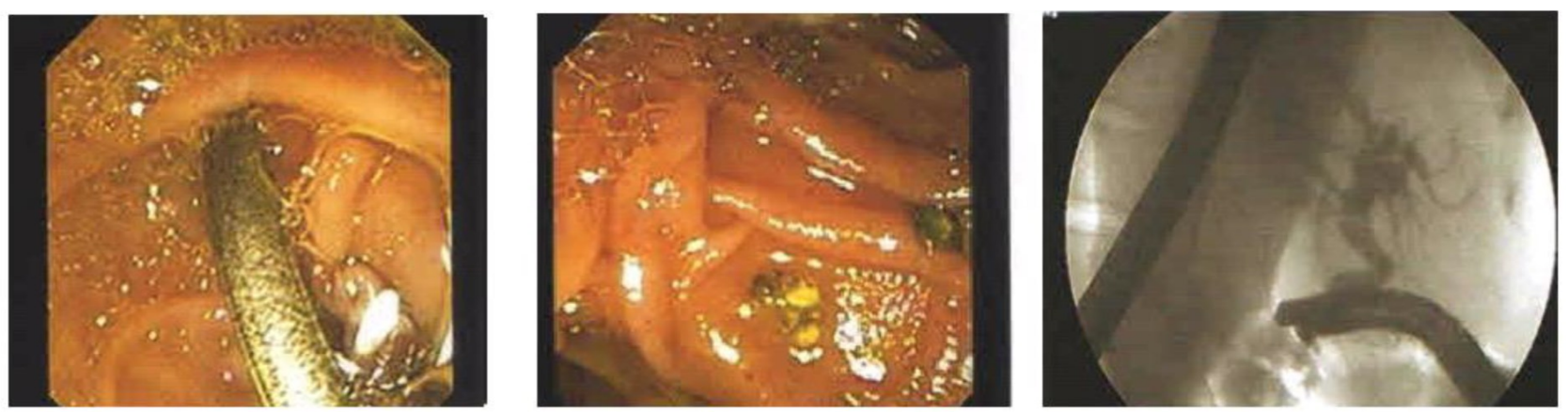

Figure 3: Images above demonstrate the removal of previously inserted stent into CBD via the fistula tract. Cholangiogram demonstrated (far right image) confirms the removal of stent due to CBD clearance and absence of distal CBD dilatation.

literature review of previous studies we found that in one case report cholangiocarcinoma was associated with cholangiobronchopleural fistula. ${ }^{9}$

Finally, after 1 month follow up status post laparoscopic cholecystectomy and stenting, patient was symptom free and had complete resolution of jaundice. Direct and indirect bilirubin was normal and ascites \& abdominal pain resolved. Consequently, stent was removed (Image C) by endoscopy and patient was recommended to follow up every 3 months.

\section{Conclusion}

In summary, we have presented an atypical case of jaundice secondary CDDF along with cholangitic abscesses and fibrosis at the distal CBD (Ampulla of Vater) which was successfully diagnosed and treated with antibiotics, ERCP with stenting and prophylactic laproscopic cholecystectomy followed by stent removal. In addition, we also shed light on the pathophysiology of CDDF and different types of fistulae which will serve as an educational (Image D) resource for current and future health care professionals.

\section{References}

1. Correia M, Amonkar D, Nayak S, Menezes J. Cholecystocolic Fistula: A Diagnostic Enigma. Saudi J-Gastroenterol 2009;15(1):42-4.

2. Balent E, Plackett $\mathrm{T}$, Lin-Hurtubise $\mathrm{K}$
Cholecystocolonic Fistula. Hawaii J Med Public Health. 2012;71(6):155-7.

3. Michowitz M, Farago C, Lazarovici I, Solowiejczyk M. Choledochoduodenal fistula: a rare complication of duodenal ulcer. $A m J$ Gastroentero. 1984;79 (5):416-20.

4. Kon CEP. Cholecystocutaneous Fistula. http:// emedicine.medscape.com/article/197206overview\#a9 (accessed July 2017)

5. Chelala E, Adam E, Rizk A, Makhoul E. Conservative management of an unusual bilioduodenal fistula post laparoscopic Duodeno-Ileal Switch (SADI-S) case report. Int J Surg Case Rep 2017;(34):1-3.

6. Aziz M, Ahmed M., et. al. Choledocoduodenal fistula secondary to duodenal ulcer disease and choledocolithiasis: Report of 2 cases. JAFMC Bangladesh 2008;4(2):38-41.

7. Mallikarjunappa B, Ashish S. Choledochoduodenal Fistula: A Rare Case Report with Review of Literature. JIMSA . 2013;26(4):226.

8. Crespi M, Montecamozzo G, Foschi D. Diagnosis and Treatment of Biliary Fistulas in the Laparoscopic Era. Gastroenterol Res Pract 2016; (2016): Article ID 6293538.

9. Zhang C, Yang G, Ling Y, Chen G, Zhou T. Distal bile duct cancers complicated with cholangiobronchopleural fistula after ERCP: A case report. Oncol Lett 2014;8(4):1828-30.
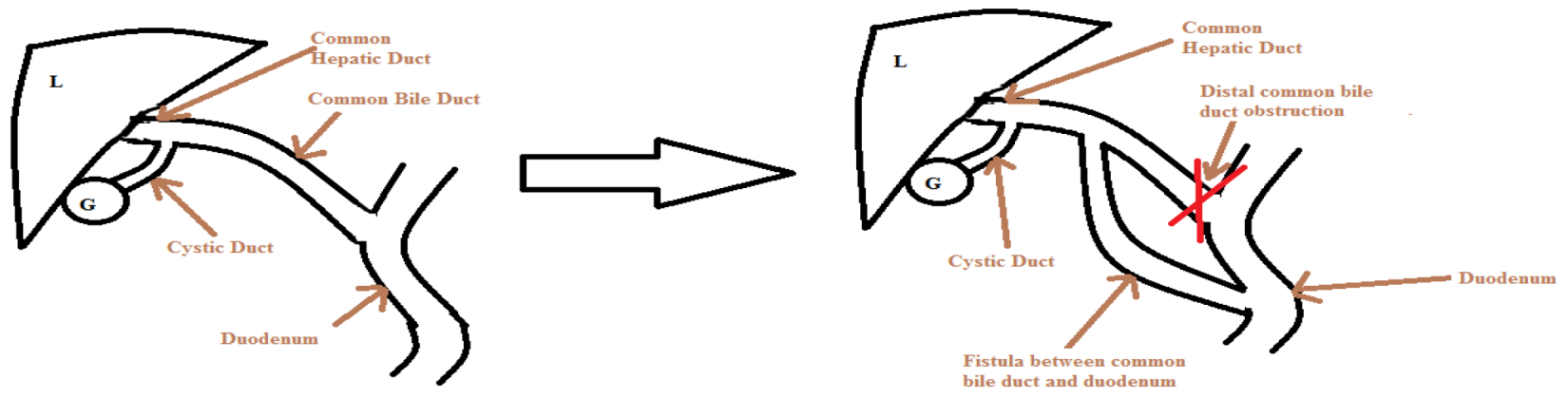

L : Liver

G: Gall Bladder

Figure 4: Picture above represents a simplistic version of the anatomy of normal vs abnormal biliary structure. On the right we can see the obstruction of the distal CBD along with abnormal fistula tract between CBD and duodenum. (Red cross in the picture on the right represents the distal CBD Ampulla of Vater where fibrosis was found). This fistula also explains why pneumobilia is present in many cases because air from the duodenum can move proximally fistula tract into the biliary system. 